\title{
ASYMPTOTICS FOR MULTILINEAR AVERAGES OF MULTIPLICATIVE FUNCTIONS
}

\author{
NIKOS FRANTZIKINAKIS AND BERNARD HOST
}

\begin{abstract}
A celebrated result of Halász describes the asymptotic behavior of the arithmetic mean of an arbitrary multiplicative function with values on the unit disc. We extend this result to multilinear averages of multiplicative functions providing similar asymptotics, thus verifying a two dimensional variant of a conjecture of Elliott. As a consequence, we get several convergence results for such multilinear expressions, one of which generalizes a well known convergence result of Wirsing. The key ingredients are a recent structural result for multiplicative functions with values on the unit disc proved by the authors and the mean value theorem of Halász.
\end{abstract}

\section{BACKGROUND AND MAIN RESUlTS}

The problem of existence of the mean value

$$
M(f):=\lim _{N \rightarrow \infty} \frac{1}{N} \sum_{n=1}^{N} f(n)
$$

of a multiplicative function $f$ with values on the unit disc has been an object of intense study in analytic number theory. P. Erdös and A. Wintner [10] conjectured that if $f$ takes real values and is bounded by 1 , then the mean value $M(f)$ exists. H. Delange [4] verified this when $\sum_{p \in \mathbb{P}} \frac{1-f(p)}{p}<\infty$ in which case $M(f) \neq 0$ unless $f\left(2^{k}\right)=-1$ for every $k \in \mathbb{N}$. The proof of the Erdös-Wintner conjecture was completed by E. Wirsing [25]; building on earlier work of his [23, 24], he showed that if the previous series diverges, then $M(f)=0$. Note that the prime number theorem corresponds to the very special case of this result where $f$ is the Möbius function. The previous results were extended to complex valued multiplicative functions by a celebrated result of G. Halász [19]. In order to give the precise statement we need a definition:

Definition (Slowly-varying sequences). We say that $w: \mathbb{N} \rightarrow \mathbb{R}$ is slowly-varying if $\max _{x \leq n \leq x^{2}}|w(n)-w(x)| \rightarrow 0$ as $x \rightarrow \infty$.

The next result is due to Halász [19]. We use it in the form given in [7, Theorem 6.2] and [22, Chapter III.4].

Theorem (Halász mean value theorem). Let $f$ be a multiplicative function that takes values on the unit disc. Then there exist constants $c \in \mathbb{C}, t \in \mathbb{R}$, and a slowly-varying sequence $w: \mathbb{N} \rightarrow \mathbb{R}$ such that

$$
\frac{1}{N} \sum_{n=1}^{N} f(n)=c N^{i t} \mathrm{e}(w(N))+o_{N \rightarrow \infty}(1) .
$$

If $c \neq 0$, then $t$ is the unique real number such that $\sum_{p \in \mathbb{P}} \frac{1}{p}\left(1-\operatorname{Re}\left(f(p) p^{-i t}\right)\right)<\infty(c=0$ if no such number exists), in which case we can take $w(N)=\frac{1}{2 \pi} \sum_{p \in \mathbb{P}, p \leq N} \frac{1}{p} \operatorname{Im}\left(f(p) p^{-i t}\right)$.

2010 Mathematics Subject Classification. Primary: 11N37; Secondary: 11B30, 11K65.

Key words and phrases. Multiplicative functions, multilinear averages, correlations, Halász, Wirsing.

B. Host was partially supported by Centro de Modelamiento Matemático, Universitad de Chile. 
Remarks. - Explicit quantitative bounds exist in the case $c=0$; see for example [22, Chapter III.4, Corollary 6.3].

- If $f(n)=n^{i t}$ for some $t \neq 0$, then $\frac{1}{N} \sum_{n=1}^{N} f(n)=\frac{N^{i t}}{1+i t}+o_{N \rightarrow \infty}(1)$; hence $M(f)$ does not exist. Lending terminology from [14], the theorem of Halász implies that the mean value $M(f)$ exists and is 0 unless $f(n)$ "pretends" to be $n^{i t}$ for some $t \in \mathbb{R}$.

Our main goal is to extend the convergence result of Wirsing and the asymptotic formula of Halász to multilinear averages of multiplicative functions. Although the one dimensional multilinear averages $\frac{1}{N} \sum_{n=1}^{N} \prod_{j=1}^{\ell} f_{j}\left(k_{j} n+a_{j}\right)$, where $k_{j}, a_{j} \in \mathbb{N}$ and $f_{j}$ are multiplicative functions with modulus at most 1 , are notoriously hard to analyze, a conjecture of P. Elliott [8, 9, Conjecture I] predicts that they satisfy asymptotics similar to those in Halász's theorem. Our main results verify these asymptotics when the one dimensional affine-linear forms $k_{j} n+a_{j}, j=1, \ldots, \ell$, are replaced with higher dimensional linear forms, or affine-linear forms with pairwise independent linear parts. The setup is as follows: We are given complex valued multiplicative functions $f_{1}, \ldots, f_{\ell}$ and linear forms $L_{1}, \ldots, L_{\ell}: \mathbb{N}^{d} \rightarrow \mathbb{N}$ given by

$$
L_{j}(\mathbf{m})=\mathbf{k}_{j} \cdot \mathbf{m} \text { for some } \mathbf{k}_{j} \in \mathbb{N}^{d} .
$$

Convention. Henceforth, we assume that all multiplicative functions take values on the interval $[-1,1]$ or the unit disc depending on whether they are real or complex valued. Furthermore, with $[N]$ we denote the set $\{1, \ldots, N\}$.

We are interested in studying the asymptotic behavior of the averages

$$
\frac{1}{N^{d}} \sum_{\mathbf{m} \in[N]^{d}} \prod_{j=1}^{\ell} f_{j}\left(L_{j}(\mathbf{m})\right) .
$$

We remark that for the purposes of this article the special case where $d=2$ and $L_{j}(m, n)=m+(j-1) n, j=1, \ldots, \ell$, is essentially as hard as the general case. If the linear forms are pairwise (linearly) independent, that is, no two are rational multiples of each other, and one of the multiplicative functions is the Möbius or the Liouville function, then B. Green and T. Tao showed in [15, Proposition 9.1], modulo conjectures which were subsequently verified in [16, 17, 18], that the averages (2) converge to 0 .

Our first result is the following:

Theorem 1.1 (Asymptotic form of multilinear averages). Let $d \in \mathbb{N}, f_{1}, \ldots, f_{\ell}$ be complex valued multiplicative functions with modulus at most 1 , and $L_{1}, \ldots, L_{\ell}: \mathbb{N}^{d} \rightarrow \mathbb{N}$ be linear forms. Then there exist $c \in \mathbb{C}, t \in \mathbb{R}$, and a slowly-varying sequence $w: \mathbb{N} \rightarrow \mathbb{R}$, such that

$$
\frac{1}{N^{d}} \sum_{\mathbf{m} \in[N]^{d}} \prod_{j=1}^{\ell} f_{j}\left(L_{j}(\mathbf{m})\right)=c N^{i t} \mathrm{e}(w(N))+o_{N \rightarrow \infty}(1) .
$$

If in addition we assume that the linear forms are pairwise independen 1 , then $t=$ $\sum_{j=1}^{\ell} t_{f_{j}}$ and we can take $w=\sum_{j=1}^{\ell} w_{f_{j}}$, where for $j=1, \ldots, \ell, t_{f_{j}}$ are the real numbers and $w_{f_{j}}$ are the slowly-varying sequences defined in Theorem 2.1 below. Furthermore, the constant $c$ in (3) is 0 unless all $f_{j}$ are "pretentious", meaning, for $j=1, \ldots, \ell$ there exist $t_{j} \in \mathbb{R}$ and Dirichlet characters $\chi_{j}$ such that $\sum_{p \in \mathbb{P}} \frac{1}{p}\left(1-\operatorname{Re}\left(\chi_{j}(p) f_{j}(p) p^{-i t_{j}}\right)\right)<\infty$.

Remarks. - In a recent preprint K. Matomäki, M. Radziwitt, T. Tao [21], using techniques from [20], prove that the averages $\frac{1}{M^{\ell}} \sum_{m_{1}, \ldots, m_{\ell} \in[M]}\left|\frac{1}{N} \sum_{n \in[N]} \prod_{j=1}^{\ell} f_{j}\left(n+m_{j}\right)\right|$ converge to 0 if $M=M(N)$ increases to infinity with $N$ at an arbitrary speed and at

\footnotetext{
${ }^{1}$ We can always reduce to this case after putting together multiplicative functions evaluated at linear forms that are pairwise dependent.
} 
least one of the multiplicative functions is "non-pretentious" in a certain uniform sense. Such results are complementary to ours and rely on very different techniques? 2

- If the linear forms are pairwise independent, then Theorem 1.1 and all subsequent results remain valid with the affine-linear forms $L_{j}(\mathbf{m})+a_{j}, a_{j} \in \mathbb{Z}$, in place of the linear forms $L_{j}(\mathbf{m})$ for $j=1, \ldots, \ell$. Interestingly, this is no longer true if two of the linear forms are dependent, even if the affine-linear forms are independent; an example from [21. Appendix A] shows that there exists a non-pretentious multiplicative function $f$ such that the averages $\frac{1}{N} \sum_{n=1}^{N} f(n) \overline{f(n+1)}$ do not converge to zero.

For $\ell=3$, the asymptotic formula (3) can be proved by combining tools from discrete Fourier analysis, a result of H. Daboussi [2, 3] that provides estimates for the Fourier coefficients of a multiplicative function, and the previously mentioned asymptotic formula of Halász. For $\ell \geq 4$ classical discrete Fourier analysis tools turn out to be insufficient for the task at hand, the reason being that for general bounded sequences the modulus of the averages (2) is not controlled by the maximum modulus of the Fourier coefficients of the individual functions (thought of as functions of $\mathbb{Z}_{N}$ ). To overcome this obstacle we use a deep structural result from [11] (see Theorem 2.2) which was proved using the toolbox of "higher order Fourier analysis". For our particular needs it implies that the general multiplicative function with modulus at most 1 can be decomposed in two terms, one that is approximately periodic and another that contributes negligibly to the averages (21). The contribution of the structured component is then analyzed using an extension of Halász's asymptotic formula (see Theorem 2.1) and after some effort the outcome is the asymptotic formula (3). The details are given in Section 3 ,

Using Theorem 1.1 we deduce the following generalization of the convergence result of E. Wirsing which deals with multilinear averages of real valued multiplicative functions:

Theorem 1.2 (Wirsing's theorem for multilinear averages). Let $f_{1}, \ldots, f_{\ell}$ be real valued multiplicative functions with modulus at most 1 and $L_{1}, \ldots, L_{\ell}: \mathbb{N}^{d} \rightarrow \mathbb{N}$ be linear forms. Then the averages (2) converge as $N \rightarrow \infty$.

Remark. If the linear forms are pairwise independent, then the result remains true if we replace the assumption that the $f_{j}$ 's take real values with the assumption that the mean values $\lim _{N \rightarrow \infty} \frac{1}{N} \sum_{n=1}^{N} f_{j}(n) \chi(n)$ exist for every Dirichlet character $\chi 3$

It follows from the asymptotic formula given in Halász's theorem that if $f$ is a complex valued multiplicative function with modulus at most 1 , then the averages $\left|\frac{1}{N} \sum_{n=1}^{N} f(n)\right|$ converge as $N \rightarrow \infty$. A similar result extends to multilinear averages:

Theorem 1.3 (Convergence of the modulus). Let $f_{1}, \ldots, f_{\ell}$ be complex valued multiplicative functions with modulus at most 1 , and $L_{1}, \ldots, L_{\ell}: \mathbb{N}^{d} \rightarrow \mathbb{N}$ be linear forms. Then the modulus of the averages (2) converges as $N \rightarrow \infty$.

Interestingly, although for complex valued multiplicative functions with modulus at most 1 the multilinear averages (2) do not in general converge, we do have convergence if in (2) each multiplicative function is paired up with its complex conjugate.

\footnotetext{
${ }^{2}$ In [21] the authors treat averages taken over a short interval $[M]$ and a long interval $[N]$; key to their analysis is a correlation estimate over short intervals between multiplicative functions and linear complex exponential sequences. In [11] and in this article, the main difficulty is different, we take $M=N$ but consider averages taken over arbitrary subspaces of $\mathbb{Z}^{\ell}$ which are given in parametric form; at the heart of this analysis lies a correlation estimate of multiplicative functions with nilsequences.

3 Whether this holds can be verified using the following consequence of the mean value theorem of Halász (see [7, Theorem 6.3]): The mean value $\lim _{N \rightarrow \infty} \frac{1}{N} \sum_{n=1}^{N} f(n)$ exists if and only if either $(i)$ $\sum_{p \in \mathbb{P}} p^{-1}\left(1-\operatorname{Re}\left(f(p) p^{-i t}\right)\right)=\infty$ for every $t \in \mathbb{R}$, or $(i i) \sum_{p \in \mathbb{P}} p^{-1}(1-f(p))$ converges, or (iii) for some $t \in \mathbb{R}$ we have $\sum_{p \in \mathbb{P}} p^{-1}\left(1-\operatorname{Re}\left(f(p) p^{-i t}\right)\right)<\infty$ and $f\left(2^{k}\right)=-2^{i k t}$ for all $k \in \mathbb{N}$.
} 
Theorem 1.4. Let $f_{1}, \ldots, f_{\ell}$ be complex valued multiplicative functions with modulus at most 1 and $L_{j}, L_{j}^{\prime}: \mathbb{N}^{d} \rightarrow \mathbb{N}, j=1, \ldots, \ell$, be pairwise independent linear forms. Then the averages

$$
\frac{1}{N^{d}} \sum_{\mathbf{m} \in[N]^{d}} \prod_{j=1}^{\ell} f_{j}\left(L_{j}(\mathbf{m})\right) \cdot \overline{f_{j}}\left(L_{j}^{\prime}(\mathbf{m})\right)
$$

converge as $N \rightarrow \infty$.

We give an application of Theorem 1.4 in ergodic theory.

Theorem 1.5. Let $(X, \mathcal{X}, \mu)$ be a probability space and for $n \in \mathbb{N}$ let $T_{n}: X \rightarrow X$ be invertible measure preserving transformations that satisfy $T_{1}:=\mathrm{id}$ and $T_{m} \circ T_{n}=T_{m n}$ for every $m, n \in \mathbb{N}$. Let also $L_{j}, L_{j}^{\prime}: \mathbb{N}^{d} \rightarrow \mathbb{N}, j=1, \ldots, \ell$, be pairwise independent linear forms. Then for all $F, G \in L^{2}(\mu)$ the averages

$$
\frac{1}{N^{d}} \sum_{\mathbf{m} \in[N]^{d}} \int F\left(T_{\prod_{j=1}^{\ell} L_{j}(\mathbf{m})} x\right) \cdot G\left(T_{\prod_{j=1}^{\ell} L_{j}^{\prime}(\mathbf{m})} x\right) d \mu
$$

converge as $N \rightarrow \infty$.

Remark. The averages (44) were studied in [1] when $d=\ell=2$ and $F=G=\mathbf{1}_{A}$ with $\mu(A)>0$ in order to prove partition regularity for certain quadratic equations.

Note that Theorem 1.5 is non-trivial even for $d=2$ and $\ell=1$. Interestingly, the averages $\frac{1}{N} \sum_{n=1}^{N} \int F\left(T_{n} x\right) \cdot G(x) d \mu$ do not necessarily converge. To see this, let $T_{n} x=$ $x+\log n \bmod 1$ act on $\mathbb{T}$ with the Haar measure and take $F(x)=\mathrm{e}(x), G(x)=\mathrm{e}(-x)$. Then the ergodic averages take the form $\frac{1}{N} \sum_{n=1}^{N} n^{2 \pi i}$. Hence, they do not converge.

It is natural to ask whether we have convergence of the mean value of a multiplicative function evaluated at homogeneous polynomials that do not necessarily factor linearly.

Problem. Let $f$ be a real valued bounded completely multiplicative function and $P \in$ $\mathbb{Z}[x, y]$ be a homogeneous polynomial with values on the positive integers. Do the averages

$$
\frac{1}{N^{2}} \sum_{1 \leq m, n \leq N} f(P(m, n))
$$

converge as $N \rightarrow \infty$ ? If the multiplicative function $f$ takes complex values, does the modulus of the above averages converge as $N \rightarrow \infty$ ?

Using Theorem 1.2 we get a positive answer when $P(m, n)=\prod_{j=1}^{\ell} L_{j}(m, n)$ where $L_{j}: \mathbb{N}^{2} \rightarrow \mathbb{N}, j=1, \ldots, \ell$, are linear forms. Lastly, we remark that for $s \geq 2$ the Gowers norms $\left\|f_{N}\right\|_{U^{s}\left(\mathbb{Z}_{N}\right)}$ (defined in Section 2) of a multiplicative function $f$ with modulus at most 1 do not necessarily converge as $N \rightarrow \infty$ even if $f$ takes values in the set $\{-1,1\}$. To see this, consider the (non-completely) multiplicative function defined by $f(n)=(-1)^{n+1}$. Then $\lim _{N \rightarrow \infty}\left\|f_{2 N}\right\|_{U^{2}\left(\mathbb{Z}_{2 N}\right)}=1$ and $\lim _{N \rightarrow \infty}\left\|f_{2 N+1}\right\|_{U^{2}\left(\mathbb{Z}_{2 N+1}\right)}=1 / 2$. On the other hand, Theorem 1.4 implies that if $f$ is a complex valued multiplicative function with modulus at most 1 , then the averages

$$
\frac{1}{N^{3}} \sum_{1 \leq m, n, r \leq N} f(m) \bar{f}(m+n) \bar{f}(m+r) f(m+n+r)
$$

converge as $N \rightarrow \infty$. Note that taking the previous average over $\mathbb{Z}_{N}$ leads to $\left\|f_{N}\right\|_{U^{2}\left(\mathbb{Z}_{N}\right)}^{4}$. Theorem 1.4 also implies convergence for higher dimensional variants of such averages. 
1.1. Notation and conventions. We denote by $\mathbb{N}$ the set of positive integers and by $\mathbb{P}$ the set of prime numbers. For $d, N \in \mathbb{N}$ we let $\mathbb{Z}_{N}:=\mathbb{Z} / N \mathbb{Z},[N]:=\{1, \ldots, N\}$, $[N]^{d}=[N] \times \cdots \times[N]$. We identify $[N]$ and $\mathbb{Z}_{N}$ in the obvious way. We let e $(t):=e^{2 \pi i t}$. A linear form $L: \mathbb{N}^{d} \rightarrow \mathbb{N}$ is a function of the form $L(\mathbf{m})=\mathbf{k} \cdot \mathbf{m}$ for some $\mathbf{k} \in \mathbb{N}^{d}$. Two linear forms are independent if one is not a rational multiple of the other. With $o_{N \rightarrow \infty}(1)$ we denote a quantity that converges to 0 when $N \rightarrow \infty$ and all other implicit parameters are fixed.

Definition. A function $f: \mathbb{N} \rightarrow \mathbb{C}$ is multiplicative if $f(m n)=f(m) f(n)$ whenever $(m, n)=1$. Moreover, $f$ is completely multiplicative if this relation holds for all $m, n \in$ $\mathbb{N}$.

With $\mathcal{M}_{\mathbb{R}}, \mathcal{M}_{\mathbb{C}}, \mathcal{M}_{\mathbb{T}}$, we denote the set of multiplicative functions on $\mathbb{N}$ that take values on $[-1,1]$, the unit disc, and the unit circle correspondingly.

A Dirichlet character (denoted by $\chi$ ) is a completely multiplicative function that is periodic and not identically 0.

\section{TWO KEY INGREDIENTS}

To prove Theorem 1.1 we will use a structural result for multiplicative functions proved by the authors in [11] and the following extension of the mean value theorem of Halász which can be found in the exact form stated here in [6, Theorem 1]:

Theorem 2.1 (Halász-Delange [5, 6, 19]). Let $f \in \mathcal{M}_{\mathbb{C}}$. Then there exists a constant $t \in \mathbb{R}$ and a slowly-varying sequence $w: \mathbb{N} \rightarrow \mathbb{R}$, such that the following holds: For every $a, b \in \mathbb{N}$ there exists a constant $c=c_{f, a, b} \in \mathbb{C}$ such that

$$
\frac{1}{N} \sum_{n=1}^{N} f(a n+b)=c N^{i t} \mathrm{e}(w(N))+o_{N \rightarrow \infty}(1) .
$$

If $c_{f, a, b}=0$ for all $a, b \in \mathbb{N}$, we set $t_{f}:=0$ and $w_{f}:=0$. If $c_{f, a, b} \neq 0$ for some $a, b \in \mathbb{N}$, then $t=t_{f}$ is the unique real number for which there exists a primitive Dirichlet character $\chi=\chi_{f}$ such that $\sum_{p \in \mathbb{P}} \frac{1}{p}\left(1-\operatorname{Re}\left(\chi(p) f(p) p^{i t}\right)\right)<\infty$. Furthermore, $\chi$ is uniquely determined and we set $w_{f}(N):=\sum_{p \in \mathbb{P}, p \leq N} \frac{1}{p} \operatorname{Im}\left(\chi(p) f(p) p^{-i t}\right)$.

Remarks. - It is important that neither $t_{f}$ nor $w_{f}$ depend on a or $b$.

- It follows from (5) and the definition of $w_{f}$, that for $f \in \mathcal{M}_{\mathbb{C}}$ we have $t_{\bar{f}}=-t_{f}$ and $w_{\bar{f}}=-w_{f}$. Hence, for $f \in \mathcal{M}_{\mathbb{R}}$ we have $t_{f}=0$ and $w_{f}=0$. In this case, the averages in (5) converge for all $a, b \in \mathbb{N}$; explicit formulas for the limit appear in [1, 6].

We now turn to the structural result; in order to state it we need to introduce some notation from [11]. Given $f: \mathbb{N} \rightarrow \mathbb{C}$ and $N \in \mathbb{N}$ we let

$$
f_{N}:=f \cdot \mathbf{1}_{[N]}
$$

and whenever appropriate, identifying the interval $[N]$ with $\mathbb{Z}_{N}$, we consider $f_{N}$ as a function in $\mathbb{Z}_{N}$. By a kernel on $\mathbb{Z}_{N}$ we mean a non-negative function on $\mathbb{Z}_{N}$ with average 1. For every prime number $N$ and $\theta>0$, in [11, Section 3.3] we defined two positive integers $Q=Q(\theta)$ and $V=V(\theta)$, and for $N>2 Q V$, a kernel $\phi_{N, \theta}$ was defined as follows: The spectrum of $\phi_{N, \theta}$ is the set

$$
\Xi_{N, \theta}:=\left\{\xi \in \mathbb{Z}_{N}:\left\|\frac{Q \xi}{N}\right\|<\frac{Q V}{N}\right\}
$$

and

$$
\widehat{\phi_{N, \theta}}(\xi):= \begin{cases}1-\left\|\frac{Q \xi}{N}\right\| \frac{N}{Q V} & \text { if } \xi \in \Xi_{N, \theta} \\ 0 & \text { otherwise. }\end{cases}
$$

We recall the definition of the $U^{s}$-Gowers uniformity norms from [12]. 
Definition (Gowers norms on a cyclic group [12]). Let $N \in \mathbb{N}$ and $a: \mathbb{Z}_{N} \rightarrow \mathbb{C}$. For $s \in \mathbb{N}$ the Gowers $U^{s}\left(\mathbb{Z}_{N}\right)$-norm $\|a\|_{U^{s}\left(\mathbb{Z}_{N}\right)}$ of a is defined inductively as follows: For every $t \in \mathbb{Z}_{N}$ we write $a_{t}(n):=a(n+t)$. We let

$$
\|a\|_{U^{1}\left(\mathbb{Z}_{N}\right)}:=\left|\frac{1}{N} \sum_{n \in \mathbb{Z}_{N}} a(n)\right|
$$

and for every $s \in \mathbb{N}$ we let

$$
\|a\|_{U^{s+1}\left(\mathbb{Z}_{N}\right)}:=\left(\frac{1}{N} \sum_{t \in \mathbb{Z}_{N}}\left\|a \cdot \bar{a}_{t}\right\|_{U^{s}\left(\mathbb{Z}_{N}\right)}^{2^{s}}\right)^{1 / 2^{s+1}} .
$$

If $f$ is a function on $\mathbb{N}$, then $\left\|f_{N}\right\|_{U^{s}\left(\mathbb{Z}_{N}\right)}$ is defined by considering the function $f_{N}=$ $f \cdot \mathbf{1}_{[N]}$ as a function on $\mathbb{Z}_{N}$.

The following structural result from [11] is crucial for our study:

Theorem 2.2 (Structure theorem for multiplicative functions [11, Theorem 8.1]). Let $s \in \mathbb{N}$ and $\varepsilon>0$. Then there exists a real number $\theta>0$ and $N_{0} \in \mathbb{N}$, depending on $s$ and $\varepsilon$ only, such that for every prime $N \geq N_{0}$, every $f \in \mathcal{M}_{\mathbb{C}}$ admits the decomposition

$$
f(n)=f_{N, \mathrm{st}}(n)+f_{N, \mathrm{un}}(n), \quad \text { for every } n \in[N],
$$

where $f_{N, \text { st }}, f_{N, \text { un }}:[N] \rightarrow \mathbb{C}$ are bounded by 1 and 2 respectively and satisfy:

(i) $f_{N, \text { st }}=f_{N} * \phi_{N, \theta}$ where $\phi_{N, \theta}$ is the kernel on $\mathbb{Z}_{N}$ defined previously and the convolution product is defined in $\mathbb{Z}_{N}$;

(ii) $\left\|f_{N, \text { un }}\right\|_{U^{s}\left(\mathbb{Z}_{N}\right)} \leq \varepsilon$.

We think of $f_{N, \text { st }}$ and $f_{N \text {, un }}$ as the structured and uniform component of $f$ respectively.

From this point on we assume that $N>2 Q V$. When convenient we identify $\mathbb{Z}_{N}$ with the set $\{0, \ldots, N-1\}$ and we denote by $(a, b) \bmod N$ the set that consists of those $\xi \in \mathbb{Z}_{N}$ such that $\xi+k N \in(a, b)$ for some $k \in \mathbb{Z}$. Note that $\xi \in \Xi_{N, \theta}$ if and only if there exists $p \in \mathbb{Z}$ such that $\xi-\frac{p}{Q} N \in(-V, V) \bmod N$. Hence,

$$
\Xi_{N, \theta}=\bigcup_{p=0}^{Q-1}\left(\frac{p}{Q} N-V, \frac{p}{Q} N+V\right) \bmod N .
$$

We may choose to include or omit the endpoints of each interval (if they are integers), since for these values the Fourier transform of the kernel is 0. Hence, we can assume that

$$
\Xi_{N, \theta}=\bigcup_{p=0}^{Q-1} \Xi_{N, \theta, p}
$$

where for $p=0, \ldots, Q-1$ we have $\Xi_{N, \theta, p}:=\left\{\left\lfloor\frac{p}{Q} N\right\rfloor+j \bmod N:-V<j \leq V\right\}$. Note that for fixed $N>2 Q V$ and $\theta>0$ the sets $\Xi_{N, \theta, p}, p=0, \ldots, Q-1$, are disjoint, each of cardinality $2 V$, hence $\left|\Xi_{N, \theta}\right|=2 Q V$. Furthermore, if $N \equiv 1 \bmod Q$, then

$$
\Xi_{N, \theta, p}=\left\{\frac{p}{Q}(N-1)+j \bmod N:-V<j \leq V\right\} .
$$

Restricting $N$ to a specific congruence class $\bmod Q$ is needed in the proof of Lemma 3.3 .

\section{Proof of the main Results}

3.1. Preparatory lemmas. In what follows we use repeatedly the following simple fact: If $(w(n))$ is a slowly-varying sequence, then for every complex valued bounded sequence $(a(n))$ we have

$$
\frac{1}{N} \sum_{n=1}^{N} a(n) \mathrm{e}(w(n))=\mathrm{e}(w(N)) \cdot \frac{1}{N} \sum_{n=1}^{N} a(n)+o_{N \rightarrow \infty}(1)
$$


We start with some preliminary lemmas.

Lemma 3.1. Let $(a(n))$ be a bounded sequence of complex numbers and $(w(n))$ be a slowly-varying sequence. Suppose that there exist $c \in \mathbb{C}$ and $t \in \mathbb{R}$ such that

$$
\frac{1}{N} \sum_{n=1}^{N} a(n)=c N^{i t} \mathrm{e}(w(N))+o_{N \rightarrow \infty}(1) .
$$

Then for every $\alpha \in \mathbb{R}$ we have

$$
\frac{1}{N} \sum_{n=1}^{N} a(n) \mathrm{e}\left(n \frac{\alpha}{N}\right)=c^{\prime} N^{i t} \mathrm{e}(w(N))+o_{N \rightarrow \infty}(1)
$$

where $c^{\prime}:=c(1+i t) \int_{0}^{1} y^{i t} \mathrm{e}(\alpha y) d y$.

Proof. Without loss of generality we can assume that $c=1 /(1+i t)$.

We first claim that

$$
\frac{1}{N} \sum_{n=1}^{N} a(n) \mathrm{e}\left(n \frac{\alpha}{N}\right)=\mathrm{e}(w(N)) \cdot \frac{1}{N} \sum_{n=1}^{N} n^{i t} \mathrm{e}\left(n \frac{\alpha}{N}\right)+o_{N \rightarrow \infty}(1) .
$$

Indeed, for $n \in \mathbb{N}$ let

$$
S(n):=\sum_{k=1}^{n}\left(a(k)-k^{i t} \mathrm{e}(w(k))\right) .
$$

Since $w$ is a slowly-varying sequence and $\frac{1}{N} \sum_{n=1}^{N} n^{i t}=\frac{N^{i t}}{1+i t}+o_{N \rightarrow \infty}(1)$, we get that $\frac{1}{N} \sum_{n=1}^{N} n^{i t} \mathrm{e}(w(n))=\frac{N^{i t}}{1+i t} \mathrm{e}(w(N))+o_{N \rightarrow \infty}(1)$; hence (10) gives that $S(n) / n \rightarrow 0$ as $n \rightarrow \infty$. Using partial summation we see that the modulus of the average

$$
\frac{1}{N} \sum_{n=1}^{N}\left(a(n)-n^{i t} \mathrm{e}(w(n))\right) \mathrm{e}\left(n \frac{\alpha}{N}\right)
$$

is at most

$$
\frac{1}{N}\left(\sum_{n=2}^{N-1}|S(n)|\left|\mathrm{e}\left((n+1) \frac{\alpha}{N}\right)-\mathrm{e}\left(n \frac{\alpha}{N}\right)\right|+|S(N)|\right)+o_{N \rightarrow \infty}(1) .
$$

Let $\varepsilon>0$. Since $S(n) / n \rightarrow 0$ as $n \rightarrow \infty$ we have $|S(n)| / n \leq \varepsilon$ for every sufficiently large $n$, and thus the last expression is bounded by

$$
\frac{1}{N}\left(\sum_{n=2}^{N-1} \varepsilon n \frac{|2 \pi \alpha|}{N}+\varepsilon N\right)+o_{N \rightarrow \infty}(1) \leq(|\pi \alpha|+1) \varepsilon+o_{N \rightarrow \infty}(1) .
$$

Since $\varepsilon$ is arbitrary we get that

$$
\frac{1}{N} \sum_{n=1}^{N}\left(a(n)-n^{i t} \mathrm{e}(w(n))\right) \mathrm{e}\left(n \frac{\alpha}{N}\right)=o_{N \rightarrow \infty}(1)
$$

and the asymptotic (11) follows because $w$ is slowly-varying.

Lastly, note that

$$
\frac{1}{N} \sum_{n=1}^{N} n^{i t} \mathrm{e}\left(n \frac{\alpha}{N}\right)=N^{i t} \cdot \frac{1}{N} \sum_{n=1}^{N}\left(\frac{n}{N}\right)^{i t} \mathrm{e}\left(n \frac{\alpha}{N}\right) .
$$

Interpreting the last average as a Riemann sum we get that it converges to the integral $\int_{0}^{1} y^{i t} \mathrm{e}(\alpha y) d y$ (integration by parts shows that the integral converges). Hence,

$$
\frac{1}{N} \sum_{n=1}^{N} n^{i t} \mathrm{e}\left(n \frac{\alpha}{N}\right)=c^{\prime} \cdot N^{i t}+o_{N \rightarrow \infty}(1)
$$


where $c^{\prime}:=\int_{0}^{1} y^{i t} \mathrm{e}(\alpha y) d y$.

Combining the above we get the asserted claim.

The next lemma enables us to get asymptotics for the discrete Fourier transform of elements of $\mathcal{M}_{\mathbb{C}}$ for certain "major arc" frequencies.

Lemma 3.2. Let $f \in \mathcal{M}_{\mathbb{C}}, t=t_{f}, w=w_{f}$, be as in Theorem 2.1. Furthermore, let $Q \in \mathbb{N}, p, \xi^{\prime} \in \mathbb{Z}$, and

$$
\xi_{N}=\frac{p}{Q} N+\frac{\xi^{\prime}}{Q}, \quad N \in \mathbb{N} .
$$

Then there exists a constant $c=c_{f, p, Q, \xi^{\prime}} \in \mathbb{C}$ such that

$$
\frac{1}{N} \sum_{n=1}^{N} f(n) \mathrm{e}\left(-n \frac{\xi_{N}}{N}\right)=c N^{i t} \mathrm{e}(w(N))+o_{N \rightarrow \infty}(1) .
$$

Remark. We are going to apply this for integers $p, \xi^{\prime}, N$ such that $N \equiv 1 \bmod Q$ and $p+\xi^{\prime} \equiv 0 \bmod Q$, in which case $\xi_{N}$ is an integer.

Proof. Notice first that the left hand side in (12) is equal to

$$
\frac{1}{Q} \sum_{r=1}^{Q} \mathrm{e}\left(-r \frac{p}{Q}\right) \frac{1}{\lfloor N / Q\rfloor} \sum_{n=1}^{\lfloor N / Q\rfloor} f(Q n+r) \mathrm{e}\left(-(Q n+r) \frac{\xi^{\prime}}{Q N}\right)+o_{N \rightarrow \infty}(1) .
$$

Hence, it suffices to show that for every fixed $Q, \xi^{\prime}$, and $r \in[Q]$, we have the asserted asymptotic for the averages

$$
\frac{1}{\lfloor N / Q\rfloor} \sum_{n=1}^{\lfloor N / Q\rfloor} f(Q n+r) \mathrm{e}\left(-(Q n+r) \frac{\xi^{\prime}}{Q N}\right) .
$$

Since e $\left(-r \xi^{\prime} /(Q N)\right) \rightarrow 1$ as $N \rightarrow \infty$ it suffices to prove the asserted asymptotic for the averages

$$
\frac{1}{\lfloor N / Q\rfloor} \sum_{n=1}^{\lfloor N / Q\rfloor} f(Q n+r) \mathrm{e}\left(-n \frac{\xi^{\prime}}{N}\right)
$$

By Theorem 2.1 there exists $c=c_{f, Q, r} \in \mathbb{C}$ such that

$$
\frac{1}{N} \sum_{n=1}^{N} f(Q n+r)=c N^{i t} \mathrm{e}(w(N))+o_{N \rightarrow \infty}(1) .
$$

Using Lemma 3.1 for $a(n):=f(Q n+r)$ and that $\lfloor N / Q\rfloor^{i t}-(N / Q)^{i t} \rightarrow 0$ and $w(N)-$ $w(\lfloor N / Q\rfloor) \rightarrow 0$ as $N \rightarrow \infty$ (since $w$ is slowly-varying), we deduce the needed asymptotic for the averages (13). This completes the proof.

Next we analyze the asymptotic behavior of the averages (2) when in place of the multiplicative functions $f_{1}, \ldots, f_{\ell}$ we use their structured components given by Theorem 2.2 .

Lemma 3.3. Let $\theta>0, d, Q \in \mathbb{N}, f_{1}, \ldots, f_{\ell} \in \mathcal{M}_{\mathbb{C}}$, and $t_{f_{j}}, w_{f_{j}}, j=1, \ldots, \ell$, be as in Theorem [2.1. Let also $L_{1}, \ldots, L_{\ell}: \mathbb{N}^{d} \rightarrow \mathbb{N}$ be linear forms let $\kappa$ be twice the sum of their coefficients. For $N \in \mathbb{N}$ let $\widetilde{N}>N$ be a prime that satisfies $\widetilde{N} \equiv 1 \bmod Q$ and suppose that the limit $\beta:=\lim _{N \rightarrow \infty}(N / \widetilde{N})$ exists and is smaller than or equal to $\kappa^{-1}$. For $j=1, \ldots, \ell$, let $f_{j, \widetilde{N}, \mathrm{st}}:=f_{j, \widetilde{N}} * \phi_{\widetilde{N}, \theta}$ where $\phi_{\widetilde{N}, \theta}$ is defined by (7) and the convolution product is defined in $\mathbb{Z}_{\widetilde{N}}$. Then there exists $c \in \mathbb{C}$ such that

$$
\frac{1}{N^{d}} \sum_{\mathbf{m} \in[N]^{d}} \prod_{j=1}^{\ell} f_{j, \widetilde{N}, \mathrm{st}}\left(L_{j}(\mathbf{m})\right)=c N^{i t} \mathrm{e}(w(N))+o_{N \rightarrow \infty}(1)
$$

where $t:=\sum_{j=1}^{\ell} t_{f_{j}}$ and $w:=\sum_{j=1}^{\ell} w_{f_{j}}$. 
Proof. By the definition of $f_{j, \widetilde{N}, \text { st }}$ we have for $j=1, \ldots, \ell$ that

$$
f_{j, \widetilde{N}, \mathrm{st}}(n)=\sum_{\xi \in \Xi_{\widetilde{N}, \theta}} \widehat{f_{j, \widetilde{N}}}(\xi) \widehat{\phi_{\widetilde{N}, \theta}}(\xi) \mathrm{e}\left(n \frac{\xi}{\widetilde{N}}\right), \quad n \in[\widetilde{N}],
$$

where $\Xi_{\widetilde{N}, \theta}$ is the spectrum of $\phi_{\widetilde{N}, \theta}$ (defined in (6) ).

Recall that for $1 \leq j \leq \ell$, the linear form $L_{j}$ has non-negative integer coefficients. By hypothesis, for every $\mathbf{m} \in[N]^{d}$ we have $0 \leq L_{j}(\mathbf{m}) \leq \kappa N / 2$. On the other hand, for $N$ large enough we have $\widetilde{N} \geq \kappa N / 2$ and thus, for every $\mathbf{m} \in[N]^{d}$, we have $L_{j}(\mathbf{m}) \in[\tilde{N}]$. Therefore, the last formula holds for $n=L_{j}(\mathbf{m})$.

Since $\widetilde{N} \equiv 1 \bmod Q$ it follows from (8) and (9) that for $\widetilde{N}>2 Q V$ if $\xi \in \Xi_{\widetilde{N}, \theta}$, then $\xi$ can be uniquely represented as

$$
\xi=\frac{p}{Q} \widetilde{N}+\frac{\xi^{\prime}}{Q}
$$

for some $p \in\{0, \ldots, Q-1\}$ and $\xi^{\prime} \in \Xi_{p, \theta}^{\prime}$ where for $p=0,1, \ldots, Q-1$ we have

$$
\Xi_{p, \theta}^{\prime}:=\{-p+j Q:-V<j \leq V\} .
$$

Hence, it suffices to show that the averages (14) satisfy the asserted asymptotic when for $j=1, \ldots, \ell$ the (finite) sequence $\left(f_{j, \widetilde{N}, \mathrm{st}}(n)\right)_{n \in[\widetilde{N}]}$ in (14) is replaced by the sequence

$$
\widehat{f_{j, \widetilde{N}}}\left(\frac{p_{j}}{Q} \widetilde{N}+\frac{\xi_{j}^{\prime}}{Q}\right) \cdot \widehat{\phi_{\widetilde{N}, \theta}}\left(\frac{p_{j}}{Q} \widetilde{N}+\frac{\xi_{j}^{\prime}}{Q}\right) \cdot \mathrm{e}\left(n\left(\frac{p_{j}}{Q}+\frac{\xi_{j}^{\prime}}{Q \widetilde{N}}\right)\right), \quad n \in[\widetilde{N}],
$$

for all possible vectors $\left(p_{1}, \ldots, p_{\ell}\right),\left(\xi_{1}^{\prime}, \ldots, \xi_{\ell}^{\prime}\right)$, where $p_{j} \in\{0, \ldots, Q-1\}$ and $\xi_{j}^{\prime} \in \Xi_{p_{j}, \theta}^{\prime}$ for $j=1, \ldots, \ell$.

For $j=1, \ldots, \ell$, by Lemma 3.2 we have that there exists $c_{j}=c_{f, p_{j}, \theta, \xi_{j}^{\prime}} \in \mathbb{C}$ such that

$$
\widehat{f_{j, \widetilde{N}}}\left(\frac{p_{j}}{Q} \widetilde{N}+\frac{\xi_{j}^{\prime}}{Q}\right)=c_{j} \widetilde{N}^{i t_{f_{j}}} \mathrm{e}\left(w_{f_{j}}(\widetilde{N})\right)+o_{N \rightarrow \infty}(1)=c_{j}^{\prime} N^{i t_{f_{j}}} \mathrm{e}\left(w_{f_{j}}(N)\right)+o_{N \rightarrow \infty}(1)
$$

where $c_{j}^{\prime}:=\beta^{-i t_{f_{j}}} c_{j}$ and the last identity follows because $\lim _{N \rightarrow \infty} \frac{N}{\widetilde{N}}=\beta$ and $w_{f_{j}}$ is a slowly-varying sequence. Hence, there exists $c=c_{f, p_{1}, \ldots, p_{\ell}, \beta, \theta, \xi_{1}^{\prime}, \ldots, \xi_{\ell}^{\prime}} \in \mathbb{C}$ such that

$$
\prod_{j=1}^{\ell} \widehat{f_{j, \widetilde{N}}}\left(\frac{p_{j}}{Q} \widetilde{N}+\frac{\xi_{j}^{\prime}}{Q}\right)=c N^{i t} \mathrm{e}(w(N))+o_{N \rightarrow \infty}(1)
$$

where $t:=\sum_{j=1}^{\ell} t_{f_{j}}$ and $w:=\sum_{j=1}^{\ell} w_{f_{j}}$.

Furthermore, it follows from (7) that

$$
\widehat{\phi_{\widetilde{N}, \theta}}\left(\frac{p_{j}}{Q} \widetilde{N}+\frac{\xi_{j}^{\prime}}{Q}\right)=1-\frac{\xi_{j}^{\prime}}{Q V}, \quad \text { whenever } \widetilde{N} \geq 2 Q V \text {. }
$$

Finally, we deal with the terms e $\left(n\left(\frac{p_{j}}{Q}+\frac{\xi_{j}^{\prime}}{Q \widetilde{N}}\right)\right)$. After substituting $L_{1}(\mathbf{m}), \ldots, L_{\ell}(\mathbf{m})$ for $n$, and writing $\mathbf{m}=\left(m_{1}, \ldots, m_{d}\right)$, they give rise to averages of the form

$$
\frac{1}{N^{d}} \sum_{1 \leq m_{1}, \ldots, m_{d} \leq N} \mathrm{e}\left(\sum_{j=1}^{d} m_{j}\left(\frac{k_{j}}{Q}+\frac{l_{j}}{Q \widetilde{N}}\right)\right)
$$

for some $k_{j}, l_{j} \in \mathbb{Z}, j=1, \ldots, d$, which depend only on the coefficients of the linear forms and the set $\bigcup_{p=0}^{Q-1} \Xi_{p, \theta}^{\prime}$. If for some $j \in\{1, \ldots, \ell\}$ the integer $k_{j}$ is not divisible by $Q$, then this average converges to 0 . Otherwise, it converges to $\prod_{j=1}^{d} \int_{0}^{1} \mathrm{e}\left(l_{j} \beta s / Q\right) d s$.

Combining the above we get the asserted asymptotic (14). 
Next we state a variant of some uniformity estimates that appear in [15, Proposition 7.1]. They can be obtained using an argument similar to the one in [11, Lemma 9.6]; we present it for completeness.

Lemma 3.4 (Uniformity estimates). Let $d, \ell \in \mathbb{N}$, with $\ell \geq 3$, and $L_{j}: \mathbb{N}^{d} \rightarrow \mathbb{N}, j=$ $1, \ldots, \ell$, be linear forms such that the forms $L_{1}, L_{j}$ are independent for $j=2, \ldots, \ell$. Let $\kappa$ be twice the sum of the coefficients of the forms $L_{j}$ and $K \in \mathbb{N}$ with $K>\kappa$. For $N \in \mathbb{N}$ large enough, let $\widetilde{N}$ be a prime with $\kappa N \leq \widetilde{N} \leq K N$ and $a_{N, 1} \ldots, a_{N, \ell}:[\widetilde{N}] \rightarrow \mathbb{C}$ be arbitrary sequences bounded by 1 . Then there exist positive constants $c=c(d, \ell)$ and $C=C(d, \ell, K)$, such that

$$
\left|\frac{1}{N^{d}} \sum_{\mathbf{m} \in[N]^{d}} \prod_{j=1}^{\ell} a_{N, j}\left(L_{j}(\mathbf{m})\right)\right| \leq C\left\|a_{N, 1}\right\|_{U^{\ell-1}\left(\mathbb{Z}_{\tilde{N}}\right)}^{c}+o_{N}(1) .
$$

Proof. After putting together terms evaluated at linear forms $L_{j}, j=2, \ldots, \ell$, that are pairwise dependent we can assume that the linear forms are pairwise independent.

For $j=1, \ldots, \ell$ and $N \in \mathbb{N}$, let $\widetilde{a}_{N, j}: \mathbb{Z} \rightarrow \mathbb{C}$ be periodic of period $\widetilde{N}$ and equal to $a_{N, j}$ on the interval $[-\lceil\widetilde{N} / 2\rceil,\lfloor\tilde{N} / 2\rfloor)$. For $\mathbf{m} \in[N]^{d}$, since $\left|L_{j}(\mathbf{m})\right|<\tilde{N} / 2$, we have $a_{N, j}\left(L_{j}(\mathbf{m})\right)=\widetilde{a}_{N, j}\left(L_{j}(\mathbf{m})\right)$. Hence,

$$
\frac{1}{N^{d}} \sum_{\mathbf{m} \in[N]^{d}} \prod_{j=1}^{\ell} a_{N, j}\left(L_{j}(\mathbf{m})\right)=\left(\frac{\tilde{N}}{N}\right)^{d} \cdot \frac{1}{\widetilde{N}^{d}} \sum_{\mathbf{m} \in \mathbb{Z}_{\tilde{N}}^{d}} \mathbf{1}_{[N]^{d}}(\mathbf{m}) \prod_{j=1}^{\ell} \widetilde{a}_{N, j}\left(L_{j}(\mathbf{m})\right) .
$$

Henceforth, we work with the right hand side and assume that the linear forms $L_{j}$ and the functions $\widetilde{a}_{N, j}$ are defined on $\mathbb{Z}_{\widetilde{N}}$.

Our first goal is to remove the cut-off $\mathbf{1}_{[N] d}(\mathbf{m})$. To do this, one can follow the method in [15. Proposition 7.1], or what turns out to be somewhat simpler, follow the argument in [11, Lemma A.1]; after approximating the cut-off by a product of smoothed out cutoffs in $\mathbb{Z}_{\widetilde{N}}$ we deduce that for some $C^{\prime}=C^{\prime}(d, K)$ the modulus of the right hand side in (16) is bounded by

$$
C^{\prime} \cdot \max _{\xi \in \mathbb{Z}_{\tilde{N}}^{d}}\left|\frac{1}{\widetilde{N}^{d}} \sum_{\mathbf{m} \in \mathbb{Z}_{\tilde{N}}^{d}} \mathrm{e}\left(\frac{\mathbf{m} \cdot \xi}{\widetilde{N}}\right) \prod_{j=1}^{\ell} \widetilde{a}_{N, j}\left(L_{j}(\mathbf{m})\right)\right|^{\frac{1}{d+1}}+o_{N \rightarrow \infty}(1) .
$$

Next we estimate the averages in (17). The pairwise independence of the linear forms $L_{j}$ implies that for $\widetilde{N} \geq \kappa^{2}$ the forms $L_{j}$ on $\mathbb{Z}_{\widetilde{N}}^{d}$ are pairwise independent over $\mathbb{Z}_{\widetilde{N}}$. Using this and an iteration of the Cauchy-Schwarz inequality (see for example the argument in [15, Proposition 7.1]) we get

$$
\max _{\xi \in \mathbb{Z}_{\tilde{N}}^{d}}\left|\frac{1}{\widetilde{N}^{d}} \sum_{\mathbf{m} \in \mathbb{Z}_{\tilde{N}}^{d}} \mathrm{e}\left(\frac{\mathbf{m} \cdot \xi}{\widetilde{N}}\right) \prod_{j=1}^{\ell} \widetilde{a}_{N, j}\left(L_{j}(\mathbf{m})\right)\right| \leq\left\|\widetilde{a}_{N, 1}\right\|_{U^{\ell-1}\left(\mathbb{Z}_{\tilde{N}}\right)} .
$$

Note that the exponential terms are going to vanish in the process because $\ell \geq 3$.

Finally, we write

$$
\mathbb{Z}_{\widetilde{N}}=I_{N} \cup J_{N} \cup\{0\} \text {, where } I_{N}:=[1,\lfloor\widetilde{N} / 2\rfloor) \text { and } J_{N}:=[\lfloor\widetilde{N} / 2\rfloor, \widetilde{N}) .
$$

Note that $\left\|\mathbf{1}_{\{0\}} \cdot \widetilde{a}_{N, 1}\right\|_{U^{\ell-1}\left(\mathbb{Z}_{\tilde{N}}\right)} \rightarrow 0$ as $N \rightarrow+\infty$. Furthermore, by the proof of [11, Lemma A.1] we have

$$
\max _{I \subset \mathbb{Z}_{\widetilde{N}}}\left\|\mathbf{1}_{I_{N}} \cdot a_{N, 1}\right\|_{U^{\ell-1}\left(\mathbb{Z}_{\widetilde{N}}\right)} \leq 3\left\|a_{N, 1}\right\|_{U^{\ell-1}\left(\mathbb{Z}_{\widetilde{N}}\right)}^{1 /\left(\left(^{\ell-1}+1\right)\right.}+o_{N}(1)
$$

where the maximum is taken over all subintervals $I$ of $\mathbb{Z}_{\widetilde{N}}$. Since $\widetilde{a}_{N, 1}$ and $a_{N, 1}$ coincide on $I_{N}$, we have $\left\|\mathbf{1}_{I_{N}} \cdot \widetilde{a}_{N, 1}\right\|_{U^{\ell-1}\left(\mathbb{Z}_{\tilde{N}}\right)}=\left\|\mathbf{1}_{I_{N}} \cdot a_{N, 1}\right\|_{U^{\ell-1}\left(\mathbb{Z}_{\tilde{N}}\right)}$. For $n \in J_{N}$ we have $\widetilde{a}_{N, 1}(n)=$ 
$\widetilde{a}_{N, 1}(n-\widetilde{N})=a_{N, 1}(n-\widetilde{N})=a_{N, 1}(\widetilde{N}-n)$. The map $n \mapsto \widetilde{N}-n$ maps the interval $J_{N}$ onto the interval $J_{N}^{\prime}:=[1,\lceil\widetilde{N} / 2\rceil]$ and thus $\left\|\mathbf{1}_{J_{N}} \cdot \widetilde{a}_{N, 1}\right\|_{U^{\ell-1}\left(\mathbb{Z}_{\widetilde{N}}\right)}=\left\|\mathbf{1}_{J_{N}^{\prime}} \cdot a_{N, 1}\right\|_{U^{\ell-1}\left(\mathbb{Z}_{\tilde{N}}\right)}$. The asserted estimate now follows by combining (16)-(19).

3.2. Proof of the main results. We proceed to prove the main results of this article.

Proof of Theorem 1.1. Without loss of generality we can assume that $\ell \geq 3$. After putting together terms evaluated at linear forms that are pairwise dependent we can assume that the linear forms are pairwise independent.

Let $\varepsilon>0$. We let $\kappa$ be twice the sum of the coefficients of the forms $L_{j}$ and

$$
\delta:=\left(\frac{\varepsilon}{2 C \ell}\right)^{1 / c}
$$

where $c, C$ are as in Lemma 3.4. We use the structural result of Theorem 2.2 for this $\delta$ in place of $\varepsilon$ and for $\ell-1$ in place of $s$. We get that there exists $\theta=\theta(\varepsilon, \ell)>0$ such that for all large enough $N \in \mathbb{N}$, if $\widetilde{N}$ denotes the smallest prime such that $\widetilde{N}>\kappa N$ and $\widetilde{N} \equiv 1 \bmod Q(Q$ was introduced in Section 2 and depends only on $\theta)$, then for $j=1, \ldots, \ell$, we have the decompositions

$$
f_{j}(n)=f_{j, \widetilde{N}, \mathrm{st}}(n)+f_{j, \widetilde{N}, \text { un }}(n), \quad n \in[\widetilde{N}],
$$

where $f_{j, \widetilde{N}, \mathrm{st}}=f_{j, \widetilde{N}} * \phi_{\widetilde{N}, \theta}\left(\phi_{\widetilde{N}, \theta}\right.$ is defined by (7D) $)$ and

$$
\left\|f_{j, \widetilde{N}, \text { un }}\right\|_{U^{\ell-1}\left(\mathbb{Z}_{\widetilde{N}}\right)} \leq \delta .
$$

The prime number theorem on arithmetic progressions implies that

$$
\lim _{N \rightarrow \infty} \frac{N}{\widetilde{N}}=\frac{1}{\kappa} .
$$

We remark that the hypothesis of Lemma 3.3 are satisfied. As in the proof of this lemma, for $N$ sufficiently large we have $L_{j}(\mathbf{m}) \in[\widetilde{N}]$ for $j=1, \ldots, \ell$ and every $\mathbf{m} \in[N]^{d}$ and thus equation (20) applies for $L_{j}(\mathbf{m})$ in place of $n$.

For $N \in \mathbb{N}$, given $a_{N, 1}, \ldots, a_{N, \ell}:[\widetilde{N}] \rightarrow \mathbb{C}$ we define

$$
A_{N}\left(a_{N, 1}, \ldots, a_{N, \ell}\right):=\frac{1}{N^{d}} \sum_{\mathbf{m} \in[N]^{d}} \prod_{j=1}^{\ell} a_{N, j}\left(L_{j}(\mathbf{m})\right) .
$$

Since for $j=1, \ldots, \ell$, the functions $f_{j, \widetilde{N} \text {,un }}:[\widetilde{N}] \rightarrow \mathbb{C}$ are bounded by 2 , it follows from Lemma 3.4 and (21) that

$$
\left|A_{N}\left(a_{N, 1}, \ldots, a_{N, \ell}\right)\right| \leq \varepsilon / \ell+o_{N}(1)
$$

if $a_{N, j}=f_{j, \widetilde{N} \text {,un }}$ for some $j=1, \ldots, \ell$ and all other sequences $a_{N, j}$ are bounded by 1. Using this property, equation (20), the fact that $f_{j}, f_{j, \widetilde{N}, \mathrm{st}}$ are bounded by 1 for $j=1, \ldots, \ell$, and telescoping, we deduce that

$$
\limsup _{N \rightarrow \infty}\left|A_{N}\left(f_{1}, \ldots, f_{\ell}\right)-A_{N}\left(f_{1, \widetilde{N}, \mathrm{st}}, \ldots, f_{\ell, \widetilde{N}, \mathrm{st}}\right)\right| \leq \varepsilon
$$

Furthermore, by Lemma 3.3 we have that the limit

$$
\lim _{N \rightarrow \infty} N^{-i t} \mathrm{e}(-w(N)) A_{N}\left(f_{1, \widetilde{N}, \mathrm{st}}, \ldots, f_{\ell, \widetilde{N}, \mathrm{st}}\right)
$$

exists for $t:=\sum_{i=1}^{\ell} t_{f_{i}}$ and $w:=\sum_{i=1}^{\ell} w_{f_{i}}$. It follows that

$$
\begin{aligned}
& \limsup _{N \rightarrow \infty} \operatorname{Re}\left(N^{-i t} \mathrm{e}(-w(N)) A_{N}\left(f_{1}, \ldots, f_{\ell}\right)\right) \leq \\
& \liminf _{N \rightarrow \infty} \operatorname{Re}\left(N^{-i t} \mathrm{e}(-w(N)) A_{N}\left(f_{1}, \ldots, f_{\ell}\right)\right)+2 \varepsilon
\end{aligned}
$$


and a similar estimate holds for the imaginary parts. Since $\varepsilon$ is arbitrary and all expres-

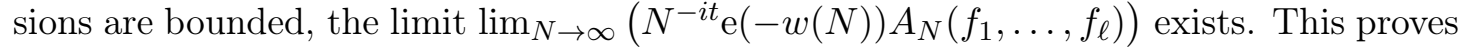
the asserted asymptotic.

Finally, we prove the last claim of Theorem 1.1. If the linear forms are pairwise independent and one of the multiplicative functions is non-pretentious, then, using the terminology of [11], it is aperiodic (see [11, Proposition 2.3]). Hence, by [11, Theorem 9.7] the averages (21) converge to 0 as $N \rightarrow \infty$.

Proof of Theorem 1.2. After putting together terms evaluated at linear forms that are pairwise dependent we can assume that the linear forms are pairwise independent. Since the multiplicative functions take real values, it follows by the definition of $t_{f}$ and $w_{f}$ in Theorem 2.1 (see the remark following this theorem) that $t_{f_{j}}=0$ and $w_{f_{j}}=0$ for $j=1, \ldots, \ell$. The result is now immediate from Theorem 1.1

Proof of Theorem 1.3. Since $\left|N^{i t} \mathrm{e}(w(N))\right|=1$ for every $N \in \mathbb{N}$ the result follows immediately from Theorem 1.1 .

Proof of Theorem 1.4. It follows from the definition of $t_{f}$ and $w_{f}$ in Theorem 2.1 that if $f \in \mathcal{M}_{\mathbb{C}}$, then $t_{\bar{f}}=-t_{f}$ and $w_{\bar{f}}=-w_{f}$. The result then follows immediately from Theorem 1.1 .

Proof of Theorem 1.5. In what follows we denote by $T F$ the composition $F \circ T$.

The polarization identity

$$
\begin{aligned}
4\left\langle T_{n} F, T_{m} G\right\rangle=\left\langle T_{n}(F+G),\right. & \left.T_{m}(F+G)\right\rangle-\left\langle T_{n}(F-G), T_{m}(F-G)\right\rangle+ \\
& i\left\langle T_{n}(F+i G), T_{m}(F+i G)\right\rangle-i\left\langle T_{n}(F-i G), T_{m}(F-i G)\right\rangle
\end{aligned}
$$

allows us to express a sequence of the form $\int T_{n} F \cdot T_{m} G d \mu, F, G \in L^{2}(\mu)$, as a linear combination of sequences of the form $\int T_{n} H \cdot T_{m} \bar{H} d \mu$ with $H \in L^{2}(\mu)$. It thus suffices to check the asserted convergence of ergodic averages when $G=\bar{F}$.

To this end, note that the action $\left(T_{n}\right)_{n \in \mathbb{N}}$ on $(X, \mathcal{X}, \mu)$ extends to a measure preserving action $\left(T_{r}\right)_{r \in \mathbb{Q}^{+}}$of the multiplicative group $\mathbb{Q}^{+}$by defining

$$
T_{a / b}:=T_{a} T_{b}^{-1} \quad \text { for all } \quad a, b \in \mathbb{N} .
$$

Let $\rho: \mathbb{Q}^{+} \rightarrow \mathbb{C}$ be defined by

$$
\rho(r):=\int T_{r} F \cdot \bar{F} d \mu, \quad r \in \mathbb{Q}^{+} .
$$

Then $\rho$ is positive definite on $\left(\mathbb{Q}^{+}, \cdot\right)$, that is, for every $n \in \mathbb{N}$, all $r_{1}, \ldots, r_{n} \in \mathbb{Q}^{+}$, and all $\lambda_{1}, \ldots, \lambda_{n} \in \mathbb{C}$, we have

$$
\sum_{i, j=1}^{n} \lambda_{i} \overline{\lambda_{j}} \rho\left(r_{i} r_{j}^{-1}\right) \geq 0 .
$$

By Bochner's theorem, there exists a unique positive finite measure $\nu$ on the dual group of the group $\mathbb{Q}^{+}$with multiplication, with a Fourier-Stieltjes transform $\widehat{\nu}$ equal to the function $\rho$. The dual group of the multiplicative group $\mathbb{Q}^{+}$is the space $\mathcal{M}_{\mathbb{T}}^{c}$ of all completely multiplicative functions of modulus 1 , the duality being given by

$$
f(m / n)=f(m) \bar{f}(n) \quad \text { for every } \quad f \in \mathcal{M}_{\mathbb{T}}^{c} \text { and every } m, n \in \mathbb{N} .
$$

The group $\mathcal{M}_{\mathbb{T}}^{c}$ is endowed with the dual topology, which is simply the compact topology of pointwise convergence.

It follows from the previous discussion that for every function $F \in L^{2}(\mu)$ there exists a positive finite measure $\nu$ on the compact Abelian group $\mathcal{M}_{\mathbb{T}}^{c}$, such that, for all $m, n \in \mathbb{N}$,

$$
\int T_{m} F \cdot \bar{T}_{n} F d \mu=\int T_{m / n} F \cdot \bar{F} d \mu=\int_{\mathcal{M}_{\mathbb{T}}^{c}} f(m / n) d \nu(f)=\int_{\mathcal{M}_{\mathbb{T}}^{c}} f(m) \bar{f}(n) d \nu(f) .
$$


Hence, in order to show convergence of the averages (4) it suffices to prove that the following averages converge

$$
\frac{1}{N^{d}} \sum_{\mathbf{m} \in[N]^{d}} \int_{\mathcal{M}_{\mathbb{T}}^{c}} f\left(\prod_{j=1}^{\ell} L_{j}(\mathbf{m})\right) \cdot \bar{f}\left(\prod_{j=1}^{\ell} L_{j}^{\prime}(\mathbf{m})\right) d \nu(f)
$$

as $N \rightarrow \infty$. Since $f$ is completely multiplicative this follows from Theorem 1.4 and the bounded convergence theorem.

\section{REFERENCES}

[1] A. Balog, A. Granville and K. Soundararajan. Multiplicative functions in arithmetic progressions. Annales mathématiques du Québec 37 (2013), 3-30.

[2] H. Daboussi. Fonctions multiplicatives presque périodiques B. D'après un travail commun avec Hubert Delange. Journées Arithmétiques de Bordeaux (Conf., Univ. Bordeaux, Bordeaux, 1974), pp. 321-324. Asterisque 24-25 (1975), 321-324.

[3] H. Daboussi and H. Delange. Quelques proprietes des functions multiplicatives de module au plus egal 1. C. R. Acad. Sci. Paris Ser. A 278 (1974), 657-660.

[4] H. Delange. Sur les fonctions arithmétiques multiplicatives. Ann. Sci. Ecole Norm. Sup. 78 (1961), 273-304.

[5] H. Delange. Sur les fonctions arithmétiques multiplicatives de module au plus égal à un. C.R. Acad. Sci. Paris Ser. A 27 (1972), 781-784.

[6] H. Delange. Sur les fonctions arithmétiques multiplicatives de module $\leq 1$. Acta Arithmetica 42 (1983), 121-151.

[7] P. Elliott. Probabilistic Number Theory I. Springer-Verlag, New York, Heidelberg, Berlin (1979).

[8] P. Elliotr. Multiplicative functions $|g| \leq 1$ and their convolutions: An overview. Séminaire de Théorie des Nombres, Paris 1987-88. Progress in Mathematics 81 (1990), 63-75.

[9] P. Elliott. On the correlation of multiplicative and the sum of additive arithmetic functions. Mem. Amer. Math. Soc. 112 (1994), no. 538.

[10] P. Erdös. Some unsolved problems. Michigan Math. J. 4 (1957), 291-300.

[11] N. Frantzikinakis and B. Host. Higher order Fourier analysis of multiplicative functions and applications. To appear in J. Amer. Math. Soc. arXiv:1403.0945.

[12] T. Gowers. A new proof of Szemerédi's theorem. Geom. Funct. Anal. 11 (2001), 465-588.

[13] A. Granville and K. Soundararajan. Decay of mean-values of multiplicative functions. Can. J. Math. 55 (2003), 1191-1230.

[14] A. Granville and K. Soundararajan. Multiplicative Number Theory: The pretentious approach. Book manuscript in preparation.

[15] B. Green and T. Tao. Linear equations in the primes. Ann. of Math. 171 (2010), 1753-1850.

[16] B. Green and T. Tao. The quantitative behaviour of polynomial orbits on nilmanifolds. Ann. of Math. 175 (2012), no. 2, 465-540.

[17] B. Green And T. TaO. The Möbius function is strongly orthogonal to nilsequences. Ann. of Math. 175 (2012), no. 2, 541-566.

[18] B. Green, T. Tao and T. Ziegler. An inverse theorem for the Gowers $U^{s+1}[N]$-norm. Ann. of Math. 176 (2012), no. 2, 1231-1372.

[19] G. Halász. Über die Mittelwerte multiplikativer zahlentheoretischer Funktionen. Acta Math. Acad. Sci. Hung. 19 (1968), 365-403.

[20] K. MatomäKi and M. RadziwiŁe. Multiplicative functions in short intervals. To appear in Ann. of Math. arXiv:1501.04585.

[21] K. Matomäki, M. Radziwile and T. TaO. An averaged form of Chowla's conjecture. Algebra 8 Number Theory 9 (2015), 2167-2196.

[22] G. Tenenbaum. Introduction à la théorie analytique et probabiliste des nombres. Cours Spécialisés, no. 1. Société Mathématique de France, Paris, 1995.

[23] E. Wirsing. Das asymptotische Verhalten von Summen uber multiplikative Funktionen. Math. Annalen 143 (1961), 75-102.

[24] E. Wirsing. Elementare Beweise des Primzahlsatzes mit Restglied. II. J. Reine Angew. Math. $\mathbf{2 1 4 / 2 1 5}$ (1964), 1-18.

[25] E. Wirsing. Das asymptotische Verhalten von Summen uber multiplikative Funktionen, II. Acta Math. Acad. Sci. Hung. 18 (1967), 411-467. 
(Nikos Frantzikinakis) University of Crete, Department of mathematics, Voutes UniverSity Campus, Heraklion 71003, Greece

E-mail address: frantzikinakis@gmail.com

(Bernard Host) Université Paris-Est Marne-la-Vallée, Laboratoire d'Analyse et de mathÉmatiques appliquées, UMR CNRS 8050, 5 Bd Descartes, 77454 Marne la Vallée Cedex, FRANCE

E-mail address: bernard.host@u-pem.fr 